\title{
Estudo toxicológico pré-clínico agudo com o extrato hidroalcoólico das folhas de Cissus sicyoides L. (Vitaceae)
}

\section{Tereza Helena Cavalcanti de Vasconcelos ${ }^{1 *}$, João Modesto-Filho ${ }^{2}$, Margareth de Fátima Formiga Melo Diniz, Hosana Bandeira Santos, Fernanda Burle de Aguiar', Patrícia Vasconcelos Leitão Moreira ${ }^{1}$}

\author{
${ }^{1}$ Departamento de Nutrição, Universidade Federal da Paraiba, Campus Universitário, \\ 58051-970, João Pessoa, PB, Brasil, \\ ${ }^{2}$ Departamento de Medicina Interna, Universidade Federal da Paraiba, Campus Universitário, \\ 58051-970, João Pessoa, PB, Brasil, \\ ${ }^{3}$ Departamento de Ciências Farmacêuticas, Universidade Federal da Paraiba, Campus Universitário, \\ 58051-970, João Pessoa, PB, Brasil, \\ ${ }^{4}$ Laboratório de Tecnologia Farmacêutica, Universidade Federal da Paraíba, Campus Universitário, \\ 58051-970, João Pessoa, PB, Brasil, \\ ${ }^{5}$ Departamento de Fisiologia e Patologia, Universidade Federal da Paraíba, Campus Universitário, \\ 58051-970, João Pessoa, PB, Brasil
}

\begin{abstract}
RESUMO: O estudo objetivou realizar ensaio toxicológico pré-clínico inicial para investigar a toxicidade da planta. $\mathrm{Na}$ investigação da $\mathrm{DL}_{50}$ foram utilizados grupos de camundongos Swiss machos $(\mathrm{n}=10)$ submetidos a doses de $5,0 \mathrm{~g} / \mathrm{kg}$, via oral, e dose de $2,0 \mathrm{~g} / \mathrm{kg}$ por via intraperitoneal, da fração aquosa das folhas (FAF) de Cissus sicyoides L. Foi retirado sangue através do plexo braquial após sete dias, para análise laboratorial de parâmetros hematológicos e bioquímicos. No estudo anatomopatológico, os animais foram examinados macro e microscopicamente, com ressecção de pulmão, coração, fígado e rins. Para análise dos dados foram utilizadas técnicas de estatística descritiva e inferencial. Na avaliação dos parâmetros hematológicos e bioquímicos dos camundongos Swiss, foram detectadas alterações nas transaminases AST e ALT e na fosfatase alcalina (FAL), caracterizando as alterações hepáticas demonstradas no estudo histopatológico. $\mathrm{Na}$ avaliação histológica do fígado de camundongos, evidenciou-se hepatite reacional com portite linfocitária crônica e lobular multifocal, hiperplasia kupferiana, colapsos focais da trama reticular, ausência de fibrose portal e lobular. O estudo toxicológico pré-clínico agudo demonstrou em camundongos alterações hepáticas.
\end{abstract}

Unitermos: Cissus sicyoides, Vitaceae, toxicidade pré-clínica.

\begin{abstract}
Acute pre-clinical toxicological study with the hydroalcoholic extract of the leaves of Cissus sicyoides L. (Vitaceae)". The aim of the study was to carry through pre-clinical toxicological assay to analyze the toxicity of the plant. In the inquiry of the $\mathrm{LD}_{50}$ groups of male Swiss rats $(\mathrm{n}=10)$ were given orally $5.0 \mathrm{~g} / \mathrm{kg}$, and $2.0 \mathrm{~g} / \mathrm{kg}$ intraperitoneally, of the water fraction of the leaves (WFL) of Cissus sicyoides L. Blood was removed through brachial plexus after seven days, for laboratory analysis of hematological and biochemical parameters. In the anatomopathological study, the animals had been examined macro and microscopically, with dissection of lung, heart, liver and kidneys. For analysis of the data techniques of descriptive and inferencial statistics had been used. In the evaluation of the hematological and biochemical parameters of the Swiss rats, alterations in aspartate aminotransferase (AST), alanine aminotransferase (ALT) and alkaline phosphatase (FAL) had been detected, characterizing the demonstrated hepatic alterations in the histopathological study. In the histological evaluation of the rats's liver, submitted to the poisoning, reactionary hepatitis with chronic linfocity portite was proven and to lobular multifocal, hiperplasia kupferiana, focal collapses of the reticular tram, absence of fibrosis, absences of cellular atipias and reactionary subcapsulite. The acute pre-clinical toxicological study demonstrated hepatic alterations in rats.
\end{abstract}

Keywords: Cissus sicyoides, Vitaceae, pre-clinical toxicity. 


\section{INTRODUÇÃO}

A família Vitaceae posssui 16 gêneros e cerca de 400 espécies lenhosas em sua maioria trepadeiras, com uma distribuição pantropical (Correa, 1926). Estudos realizados com algumas espécies de Cissus têm revelado várias atividades farmacológicas, dentre as quais destacam-se as atividades: antioxidante e antimicrobiana (Murthy et al., 2003; Silva et al., 2007), inibidor da enzima acetilcolinesterase (Barbosa-Filho et al., 2006), hipoglicemiante (Barbosa et al., 2002), na prevenção da osteoporose (Shirwaikar et al., 2003), entre outras.

A espécie vegetal Cissus sicyoides L. é conhecida popularmente como "anil trepador", "cipópucá", "cortina japonesa", "uva brava" e insulina. As folhas são empregadas externamente contra o reumatismo, a cura de abscessos e a infusão das folhas e do caule utilizadas na inflamação muscular, epilepsias, derrame cerebral, hipertensão e ativadora da circulação sanguínea. Recentemente, esta espécie vem sendo muito empregada pela população para o tratamento de diabetes, sendo por isso conhecida como "insulina" e motivo para estudos botânicos, químicos e farmacológicos no Brasil e no exterior (Beltrame et al., 2001; Pepato et al., 2003; Barbosa-Filho et al., 2005).

$\mathrm{Na}$ Cissus sicyoides encontram-se compostos químicos como: cianidina, cianidina-3-O-arabinose, cianidina-3-ramnosil-arabinosideo, delfinidina, delfinidina-3-O-beta-D-glucosideo, delfinidina-3-Obeta-D-rutinosideo, delfinidina-3-ramnosideo, todos pertencentes ao grupo dos flavonóides, além de caroténos (Beltrame et al., 2001; Silva et al., 1996).

Estudo etnofarmacológico de Cissus sicyoides L. na cidade de João Pessoa - Paraíba (Vasconcelos et al., 2002), evidenciou que a população usa as folhas, nas formas de preparações: infusão e decocção. Preparações de infusão foram realizadas, com 1,0 $\mathrm{g}$ do pó das folhas para $150 \mathrm{~mL}$ de água. Foi determinado o peso seco de várias amostras de $1,0 \mathrm{~mL}$, e o material sólido obtido, pesou em média $1,8 \mathrm{mg}$ de 10 preparações, ou seja 1,0 $\mathrm{mL}$, dos preparados, continha cerca de $1,8 \mathrm{mg}$ de sólidos. Como o ser humano (peso médio dos voluntários do estudo 60 quilos) bebe cerca de $150 \mathrm{~mL}$ (1 xícara) deste infuso/dia, conclui-se que a dose de uso popular seria de $4,5 \mathrm{mg} / \mathrm{kg} / \mathrm{dia}$.

Considerando que o uso de plantas é prática prevalente em ampla faixa populacional brasileira, especialmente onde as condições sócio-econômicas são mais precárias, bem como o uso em abundância desta planta pela referida população, este estudo objetivou investigar a toxicidade pré-clínica com as folhas de Cissus sicyoides, obtidas no horto de plantas medicinais do LTF/UFPB, onde a planta é cultivada.

\section{MATERIAL E MÉTODOS}

\section{Local da pesquisa}

O material botânico utilizado nos experimentos foram as folhas de Cissus sicyoides L., obtido do Horto de Plantas Medicinais do Laboratório de Tecnologia Farmacêutica (LTF) da Universidade Federal da Paraíba (UFPB), onde a planta é cultivada.

As folhas foram desidratadas em estufa com ar circulante, a $38^{\circ} \mathrm{C}$, por 72 horas e trituradas em moinho tipo Harley. Depois foram maceradas com etanol a 35 $\%$ a temperatura ambiente, por 72 horas. Em seguida, o material foi filtrado e submetido a uma nova extração por igual período. Os filtrados foram concentrados a vácuo em rotavapor, fornecendo um material escuro, higroscópico, denominado extrato hidroalcoólico bruto (EHAB), que foi submetido a liofilização. Quando requerido, o EHAB era dissolvido em água, filtrado, resultando na fração aquosa das folhas (FAF), cujo resíduo sólido, correspondente a $90 \%$ do $\mathrm{EHAB}$, e se constituiu na matéria-prima para os ensaios toxicológicos pré-clínicos realizados.

As atividades da pesquisa pré-clínica foram realizadas no Laboratório de Toxicologia e Farmacologia e no Biotério, do Núcleo de Pesquisas em Produtos Naturais do LTF/UFPB, e Hospital Universitário Lauro Wanderley. Também foram desenvolvidas as análises anatomopatológicas, no Instituto de Anatomia Patológica e Citologia (IAPCI), no município de João Pessoa - PB.

\section{Investigação da $\mathrm{DL}_{50}$}

Grupos de camundongos Swiss $(\mathrm{n}=10)$ machos, colocados 5 animais por caixa, foram submetidos a doses de $5,0 \mathrm{~g} / \mathrm{kg}$, via oral (gavagem), e dose de 2,0 g/ $\mathrm{kg}$ por via intraperitoneal, da fração aquosa das folhas (FAF) de Cissus sicyoides. Estas doses são consideradas máximas e letais segundo a Resolução 17/2000 (Anvisa, 2000).

Quando ocorreram, foi contabilizado o número de animais eventualmente mortos, para a determinação da dose responsável pela morte de $50 \%$ dos animais experimentais $\left(\mathrm{DL}_{50}\right)$, com limite de confiança de $95 \%$, segundo o método de Litchfield e Wilcoxon (1949), no qual se conta o número de animais mortos para cada grupo e se calcula o percentual, que transformado em probitos é aplicado na ordenada do gráfico, ficando o logarítimo da dose de FAF na abscissa.

\section{Análise laboratorial do sangue}

Sete dias após a administração das doses utilizadas por via oral e intraperitoneal, dos animais que não morreram na investigação da $\mathrm{DL}_{50}$, foi retirado sangue através do plexo braquial, para análise laboratorial de parâmetros hematológicos, estudo das séries vermelha (eritrograma) e série branca (leucograma) e contagem 
de plaquetas. Estas determinações foram realizadas no analisador celular COBAS ARGOS $50^{\circledR}$ - ROCHE. Os esfregaços sanguíneos foram corados automaticamente no HEMATEL 200 e analisados em microscópio Olympus, para confirmação e controle da contagem das células (Angelucci; Mantovani, 1986).

A análise laboratorial de parâmetros bioquímicos foi realizada em amostras de soro. As dosagens da glicose, triglicerídios, colesterol total, aspartato transaminase (AST), alanina transaminase (ALT), fosfatase alcalina (FAL), proteínas totais, uréia, creatinina, ácido úrico foram realizadas, no analisador bioquímico automatizado, COBAS MIRA PLUS ${ }^{\circledR}$ - ROCHE. O íons sódio, potássio e cloreto foram determinados pelo analisador de íons seletivos BAYER, utilizando-se método eletrodo íon seletivo (Aroch et al., 1999).

\section{Estudo anatomopatológico}

Também, após 7 dias da administração da dose utilizada pelas vias oral e intraperitoneal, nos animais para investigação da $\mathrm{DL}_{50}, 30 \%$ dos animais foram sacrificados por descolamento cervical, examinados macroscopicamente, com ressecção de pulmão, coração, fígado e rins. As secções teciduais dos órgãos excisados, fixados em formalina (solução de formol a $10 \%$ ) tamponada, após 24 horas, forma resseccionadas para processamento histopatológico: desidratação com séries crescentes de álcool (70 a 100\%), diafanização em xilol, impregnação e inclusão em parafina, segundo os métodos habituais (Bacha; Wood, 1990). Em micrótomo, os fragmentos tissulares foram seccionados em espessura de 3,0 $\mu \mathrm{M}$ e subseqüentemente submetidos a coloração hematoxilina-eosina e tricrômico de Masson, e examinados ao microscópio óptico.

\section{RESULTADOS}

\section{Investigação da $\mathrm{DL}_{50}$}

No ensaio para determinação do grau de toxicidade - investigação da dose letal $50 \%\left(\mathrm{DL}_{50}\right)$, apresentado pela fração aquosa das folhas (FAF) de Cissus sicyoides, observou-se que não houve mortes nas duas vias de administração.

\section{Análise laboratorial do sangue}

Sete dias após administração das doses de 2,0 g/ $\mathrm{kg}$ via i.p. e 5,0 g/kg v.o. em camundongos Swiss, foram detectados, alterações dos parâmetros bioquímicos e parâmetros hematológicos.

Após a administração da dose de $2,0 \mathrm{~g} / \mathrm{kg}$ via i.p, foram evidenciadas alterações estatisticamente significativas em alguns parâmetros hematológicos (hematócrito, VCM, CHCM, monócitos e plaquetas) e em alguns parâmetros bioquímicos (AST, ALT, fosfatase alcalina, ácido úrico, sódio e potássio).

Após a administração da dose de $5,0 \mathrm{~g} / \mathrm{kg}$ via oral, foram evidenciadas alterações estatisticamente significativas em alguns parâmetros hematológicos (hemoglobina, VCM, CHCM, neutrófilos, monócitos e plaquetas) e em alguns parâmetros bioquímicos (AST, ALT, fosfatase alcalina, triglicerídios, ácido úrico, uréia, proteínas totais e sódio) conforme demonstram as tabelas 1 e 2 .

As alterações foram caracterizadas pelo aumento significativo dos níveis séricos de AST, ALT, fosfatase alcalina, ácido úrico e sódio, em relação aos valores de referência (resultados obtidos antes e após a administração das doses). Essas mudanças significativas de AST, ALT e fosfatase alcalina, que induziram a alterações na função hepática são confirmadas no estudo anatomopatológico, descrito abaixo.

Para os parâmetros nível de potássio sérico e valor do hematócrito, o aumento foi significativo, apenas na dose de 2,0 g/ $\mathrm{kg}$ i.p. Quanto as variáveis $\mathrm{VCH}$ e uréia, os aumentos foram significativos apenas na dose de 5,0 $\mathrm{g} / \mathrm{kg}$ via oral, enquanto para os triglicerídios, proteínas totais, hemoglobina, neutrófilos existiram diminuições significativas.

\section{Estudo anatomopatológico}

No estudo anatomopatológico em camundongos Swiss submetidos a testes de investigação de toxicidade aguda, com administração de doses de $5,0 \mathrm{~g} / \mathrm{kg}$ v.o. e 2,0 $\mathrm{g} / \mathrm{kg}$ i.p., da fração aquosa das folhas (FAF) de Cissus sicyoides, foram examinados os seguintes órgãos: fígado, pulmões, rins e coração.

No exame anatomopatológico de cortes tissulares do estudo por via oral e intraperitoneal, permaneceram dentro dos parâmetros de normalidade os órgãos a seguir: coração, rins e pulmões. O fígado foi o único órgão que sofreu alterações na dose de $5,0 \mathrm{~g} / \mathrm{kg}$ v.o e na dose $2,0 \mathrm{~g} / \mathrm{kg}$ i.p, descritas nos quadros 1 e 2 e ilustradas nas figuras 1,2 e 3 .

\section{DISCUSSÃO}

O extrato hidroalcoólico bruto (EHA) (rendimento de $19 \%$ ) e fração aquosa das folhas de Cissus sicyoides (rendimento de $90 \%$ do EHA) foram obtidos para realização dos ensaios pré-clínicos. Geralmente o rendimento dos extratos obtidos à partir das plantas varia de acordo com fatores relacionados com a planta e com os métodos de extração e líquidos extratores utilizados (Diniz, 2000).

Com o intuito de melhor investigar aspectos toxicológicos da Cissus sicyoides foram realizados ensaios pré-clínicos de toxicidade aguda, contribuindo com os estudos da Cissus sicyoides, como alimento com alegação de propriedade de saúde, utilizando o roteiro da Portaria 116/96 (Secretaria de Vigilância Sanitária, 
1996), com algumas modificações, uma vez que as Resoluções 17/99, 18/99, 19/99 da Anvisa (Anvisa, 1999a,b,c) não estabelecem roteiros para a realização de protocolos experimentais pré-clínicos, apenas citam que estes devem ser realizados.

A avaliação farmacológica pré-clínica do potencial antidiabético das folhas de Cissus sicyoides já foi investigada e detectada por vários autores, como Beltrame et al. (2001) e Pepato et al. (2003).

Apenas um estudo é citado pela literatura com o objetivo de investigar em ratos o efeito tóxico do decocto de folhas de Cissus sicyoides a nível hepático, muscular e de vasos sanguíneos, o qual demonstrou que o decocto apresentou toxicidade a nível hepático detectado por aumento de aspartato transaminase (AST) e alanina transaminase (ALT), mas apenas quando associado ao diabetes. Os demais órgãos avaliados não sofreram efeito tóxico do decocto (Pepato et al., 2000).

No ensaio toxicológico pré-clínico agudo, realizado para avaliar o efeito tóxico do extrato hidroalcoólico de Cissus sicyoides em camundongos, demonstrou que a fração aquosa não induziu a morte de nenhum animal, quando estes foram submetidos às doses máximas estipuladas $(5,0 \mathrm{~g} / \mathrm{kg}$ v.o. - dose 1.111 vezes a do uso popular e $2,0 \mathrm{~g} / \mathrm{kg}$ i.p. - dose 444 vezes a do uso popular) por via oral e intraperitoneal respectivamente, salientando-se a não existência de estudos prévios para comparação.

$\mathrm{Na}$ avaliação dos parâmetros hematológicos e bioquímicos dos camundongos Swiss, submetidos aos ensaios toxicológicos pré-clínico, tanto por via oral como intraperitoneal, foram detectadas aumentos nas transaminases AST e ALT e na fosfatase alcalina (FAL), caracterizando as alterações hepáticas demonstradas no estudo histopatológico, corroborando o estudo de Pepato et al. (2000).

Também foram detectados aumentos significativos da uréia (após $5,0 \mathrm{~g} / \mathrm{kg}$ v.o.), ácido úrico e sódio (após $2,0 \mathrm{~g} / \mathrm{kg}$ i.p. e $5,0 \mathrm{~g} / \mathrm{kg}$ v.o.) e potássio (após $2,0 \mathrm{~g} / \mathrm{kg}$ i.p.).

Comumente, na clínica dos pequenos animais, são detectadas anormalidades nas atividades séricas

Tabela 1. Parâmetros hematológicos obtidos do soro de camundongos Swiss, tratados com $2,0 \mathrm{~g} / \mathrm{kg}$ via intraperitoneal e $5,0 \mathrm{~g} / \mathrm{kg}$ via oral, da fração aquosa (FAF) de Cissus sicyoides, após sete dias (toxicidade aguda).

\begin{tabular}{l|c|c|c|c}
\hline \multirow{2}{*}{ Parâmetros } & \multicolumn{3}{|c|}{ Tratamento } & \multirow{2}{*}{ Valor de $\mathrm{p}$} \\
\cline { 2 - 5 } & Basal & $\begin{array}{c}\text { Após } \\
2,0 \mathrm{~g} / \mathrm{Kg} \mathrm{i.p.}\end{array}$ & $\begin{array}{c}\text { Após } \\
5,0 \mathrm{~g} / \mathrm{Kg} \text { v.o }\end{array}$ & $\begin{array}{c}(1) \mathrm{p}=0,234 \\
(2) \mathrm{p}=0,267\end{array}$ \\
\hline Hemácias $\left(10^{6} / \mathrm{mm}^{3}\right)$ & $8,66 \pm 0,36$ & $10,04 \pm 1,01$ & $7,19 \pm 1,17$ & $\begin{array}{c}(1) \mathrm{p}=0,858 \\
(2) \mathrm{p}=0,049^{*}\end{array}$ \\
\hline Hemoglobina $(\mathrm{g} / \mathrm{dl})$ & $13,36 \pm 1,10$ & $13,46 \pm 0,49$ & $11,96 \pm 0,31$ & $\begin{array}{c}(1) \mathrm{p}=0,015^{*} \\
(2) \mathrm{p}=0,769\end{array}$ \\
\hline Hematócrito (\%) & $40,76 \pm 1,35$ & $46,00 \pm 1,01$ & $41,28 \pm 1,04$ & $\begin{array}{c}(1) \mathrm{p}=0,004^{*} \\
(2) \mathrm{p}=0,009^{*}\end{array}$ \\
\hline VCM $\left(\mu^{3}\right)$ & $48,60 \pm 1,32$ & $54,32 \pm 0,46$ & $54,00 \pm 0,85$ & $\begin{array}{c}(1) \mathrm{p}=0,508 \\
(2) \mathrm{p}=0,884\end{array}$ \\
\hline HCM $(\mu \mu \mathrm{g})$ & $15,56 \pm 0,51$ & $15,94 \pm 0,18$ & $15,66 \pm 0,41$ & $\begin{array}{c}(1) \mathrm{p}=0,001^{*} \\
(2) \mathrm{p}=0,001^{*}\end{array}$ \\
\hline CHCM (\%) & $32,54 \pm 0,55$ & $28,98 \pm 0,48$ & $29,02 \pm 0,38$ & $\begin{array}{c}(1) \mathrm{p}=0,784 \\
(2) \mathrm{p}=0,655\end{array}$ \\
\hline Leucócitos $\left(10^{3} / \mathrm{mm}^{3}\right)$ & $8,16 \pm 1,08$ & $8,47 \pm 0,20$ & $7,65 \pm 0,19$ & $\begin{array}{c}(1) \mathrm{p}=0,292 \\
(2) \mathrm{p}=0,018^{*}\end{array}$ \\
\hline Neutrófilos $(\%)$ & $18,40 \pm 1,94$ & $22,80 \pm 3,38$ & $12,00 \pm 2,12$ & $\begin{array}{c}(1) \mathrm{p}=0,094 \\
(2) \mathrm{p}=0,083\end{array}$ \\
\hline Eosinófilos $(\%)$ & $0,36 \pm 0,05$ & $0,18 \pm 0,02$ & $0,16 \pm 0,01$ & $\begin{array}{c}(1) \mathrm{p}=1,000 \\
(2) \mathrm{p}=0,080\end{array}$ \\
\hline Linfócitos $(\%)$ & $77,60 \pm 3,70$ & $77,60 \pm 1,43$ & $67,60 \pm 3,32$ & $\begin{array}{l}(1) \mathrm{p}=0,001^{*} \\
(2) \mathrm{p}=0,020^{*}\end{array}$ \\
\hline Monócitos $(\%)$ & $2,00 \pm 0,35$ & $4,40 \pm 0,24$ & $4,40 \pm 0,67$ & $\begin{array}{l}(1) \mathrm{p}=0,000^{*} \\
(2) \mathrm{p}=0,000^{*}\end{array}$ \\
\hline Plaquetas $\left(10^{3} / \mathrm{mm}^{3}\right)$ & $254,00 \pm 17,20$ & $879,00 \pm 8,79$ & $794,60 \pm 54,00$ & \\
\hline
\end{tabular}

Os valores estão expressos em média \pm EPM $n=5$ animais (camundongos machos Swiss). Teste t de Student a $5 \%$ de probabilidade.

(1) Valor relativo à comparação entre o basal e o tratamento com a dose $2,0 \mathrm{~g} / \mathrm{kg}$ i.p.

(2) Valor relativo à comparação entre o basal e o tratamento com a dose $5,0 \mathrm{~g} / \mathrm{kg}$ v.o.

(*) Diferença significante ao nível de 5,0\%. 
Tabela 2. Parâmetros bioquímicos obtidos do soro de camundongos Swiss, tratados com 2,0 g/kg via intraperitoneal e 5,0 g/kg via oral, da fração aquosa (FAF) de Cissus sicyoides, após sete dias (toxicidade aguda).

\begin{tabular}{|c|c|c|c|c|}
\hline \multirow[b]{2}{*}{ Parâmetros } & \multicolumn{3}{|c|}{ Tratamento } & \multirow[b]{2}{*}{ Valor de $\mathrm{p}$} \\
\hline & Basal & $\begin{array}{l}\text { Após (7 dias) } \\
2,0 \mathrm{~g} / \mathrm{kg} \text { i.p. }\end{array}$ & $\begin{array}{c}\text { Após (7 dias) } \\
5,0 \mathrm{~g} / \mathrm{kg} \text { v.o }\end{array}$ & \\
\hline Glicose (mg/dL) & $89,80 \pm 1,53$ & $94,60 \pm 2,01$ & $93,40 \pm 1,81$ & $\begin{array}{l}\text { (1) } \mathrm{p}=0,094 \\
\text { (2) } \mathrm{p}=0,167\end{array}$ \\
\hline Colesterol (mg/dL) & $80,00 \pm 3,52$ & $87,60 \pm 2,71$ & $89,4 \pm 3,98$ & $\begin{array}{l}\text { (1) } p=0,126 \\
\text { (2) } p=0,115\end{array}$ \\
\hline Triglicerídios (mg/dL) & $107,00 \pm 6,76$ & $111,20 \pm 11,43$ & $72,40 \pm 4,58$ & $\begin{array}{l}\text { (1) } \mathrm{p}=0,760 \\
\text { (2) } \mathrm{p}=0,003^{*}\end{array}$ \\
\hline AST (U/L) & $77,20 \pm 8,65$ & $155,00 \pm 4,89$ & $212,80 \pm 10,54$ & $\begin{array}{l}\text { (1) } \mathrm{p}=0,000^{*} \\
\text { (2) } \mathrm{p}=0,000^{*}\end{array}$ \\
\hline ALT (U/L) & $50,60 \pm 6,14$ & $67,40 \pm 2,68$ & $89,20 \pm 3,04$ & $\begin{array}{l}\text { (1) } \mathrm{p}=0,036^{*} \\
\text { (2) } \mathrm{p}=0,000^{*}\end{array}$ \\
\hline Fosfatase alcalina (U/L) & $149,40 \pm 16,99$ & $212,20 \pm 12,13$ & $228,00 \pm 10,78$ & $\begin{array}{l}\text { (1) } \mathrm{p}=0,017^{*} \\
\text { (2) } \mathrm{p}=0,005^{*}\end{array}$ \\
\hline Proteínas totais $(\mathrm{mg} / \mathrm{dL})$ & $6,16 \pm 0,22$ & $5,84 \pm 0,13$ & $5,16 \pm 0,19$ & $\begin{array}{l}\text { (1) } \mathrm{p}=0,252 \\
\text { (2) } \mathrm{p}=0,003^{*}\end{array}$ \\
\hline Uréia (mg/dL) & $57,60 \pm 2,54$ & $62,40 \pm 2,80$ & $91,00 \pm 9,36$ & $\begin{array}{l}\text { (1) } \mathrm{p}=0,240 \\
\text { (2) } \mathrm{p}=0,009^{*}\end{array}$ \\
\hline Ácido úrico $(\mathrm{mg} / \mathrm{dL})$ & $2,00 \pm 0,17$ & $5,70 \pm 0,43$ & $3,96 \pm 0,22$ & $\begin{array}{l}\text { (1) } \mathrm{p}=0,000^{*} \\
\text { (2) } \mathrm{p}=0,000^{*}\end{array}$ \\
\hline Creatinina $(\mathrm{mg} / \mathrm{dL})$ & $0,34 \pm 0,02$ & $0,40 \pm 0,03$ & $0,30 \pm 0,01$ & $\begin{array}{l}\text { (1) } p=0,347 \\
\text { (2) } p=0,524\end{array}$ \\
\hline Sódio $(\mathrm{mEq} / \mathrm{l})$ & $140,40 \pm 2,29$ & $151,40 \pm 1,14$ & $155,40 \pm 3,08$ & $\begin{array}{l}\text { (1) } \mathrm{p}=0,002^{*} \\
\text { (2) } \mathrm{p}=0,004^{*}\end{array}$ \\
\hline Potássio $(\mathrm{mEq} / \mathrm{L}$ & $4,96 \pm 0,52$ & $6,38 \pm 0,27$ & $4,96 \pm 0,40$ & $\begin{array}{l}\text { (1) } \mathrm{p}=0,043^{*} \\
\text { (2) } \mathrm{p}=1,000\end{array}$ \\
\hline Cloreto $(\mathrm{mEq} / \mathrm{L})$ & $105,60 \pm 1,83$ & $110,80 \pm 1,53$ & $110,80 \pm 1,53$ & $\begin{array}{l}\text { (1) } \mathrm{p}=0,061 \\
\text { (2) } \mathrm{p}=0,061\end{array}$ \\
\hline
\end{tabular}

Os valores estão expressos em média \pm EPM $n=5$ animais (camundongos machos Swiss). Teste t de Student a $5 \%$ de probabilidade.

(1) Valor relativo à comparação entre o basal e o tratamento com a dose $2,0 \mathrm{~g} / \mathrm{kg}$ i.p.

(2) Valor relativo à comparação entre o basal e o tratamento com a dose 5,0 g/kg v.o.

(*) Diferença significante ao nível de 5,0\%.

das enzimas hepáticas, as quais são consideradas indicadores sensíveis de perturbações hepatobiliares. Elevações nas atividades sérico enzimáticas podem dever-se a alterações reversíveis ou irreversíveis na permeabilidade celular, indução de enzimas microssomais ou lesões estruturais associadas à necrose, colestase ou isquemia hepatocelular. Diversos processos patológicos envolvendo o fígado podem causar elevações proporcionalmente distintas nas enzimas hepáticas, devido à variação na distribuição de cada enzima específica no lóbulo hepático (Sharon; Center, 1995).

As transaminases são enzimas que catalisam a interconversão de aminoácidos e alfa-ceto-ácidos por transferência do grupo amino. Estão amplamente distribuídas nos tecidos, predominando AST, no fígado, coração, músculo cardíaco, músculo estriado, rim e pâncreas, e ALT no fígado, rim e coração. A AST está presente no citosol e na mitocôndria dos hepatócitos. A atividade plasmática desta enzima é controlada por um mecanismo de liberação enzimática localizada na membrana do hepatócito. A velocidade de excreção das transaminases é variável, a AST elimina-se mais rapidamente do que a ALT (Miller; Gonçalves, 1999).

A fosfatase alcalina (FAL) é uma enzima fosfohidrolase, encontrada em vários tecidos, com maiores concentrações no fígado, no epitélio do trato biliar e nos ossos. No fígado, a FAL é secretada pelos hepatócitos e pelas células da mucosa do trato biliar. Geralmente qualquer hepatopatia ativa pode aumentar os valores de FAL, mas as maiores elevações nos níveis da enzima ocorrem nos casos de obstrução do trato biliar. Nos casos de drogas hepatotóxicas, as elevações da enzima são menores (Miller; Gonçalves, 1999).

$\mathrm{Na}$ avaliação histológica do fígado de camundongos, submetidos à $(5 \mathrm{~g} / \mathrm{kg})$ via oral e $(2 \mathrm{~g} /$ $\mathrm{kg}$ ) via intraperitoneal, da FAF de Cissus sicyoides, evidenciou-se hepatite reacional com portite linfocitária crônica e lobular multifocal, hiperplasia kupferiana, colapsos focais da trama reticular. 
Quadro 1. Exame anatomopatológico do fígado do animal submetido à 5,0 g/kg v.o. de FAF de Cissus sicyoides.

\begin{tabular}{|c|c|}
\hline \multicolumn{2}{|c|}{ Órgão: Fígado } \\
\hline \multicolumn{2}{|c|}{$\begin{array}{c}\text { Material: Fragmentos teciduais, irregulares. } \\
\text { Medida: O maior mede } 1,2 \times 0,7 \mathrm{~cm} \text {; o menor mede } 0,5 \times 0,2 \mathrm{~cm} \text {. }\end{array}$} \\
\hline Macroscopia & Microscopia \\
\hline $\begin{array}{l}\text { A superfície externa do material examinado é cinza, } \\
\text { lisa. }\end{array}$ & 1. Arquitetura do tipo lobular. \\
\hline \multirow{4}{*}{$\begin{array}{l}\text { Superfície de corte compacta. Seções representativas } \\
\text { encaminhadas para exame são submetidas às } \\
\text { seguintes colorações: hematoxilina-eosina, tricrômico } \\
\text { de Masson, picro-sirius e método de Gordon e Sweet } \\
\text { (reticulina), além de Perls, para detecção de } \\
\text { sobrecarga férrica. }\end{array}$} & $\begin{array}{l}\text { 2. Espaços portais com tríades vásculo-biliares sem } \\
\text { particularidades histológicas, apresentando ocasional } \\
\text { alargamento inflamatório à custa de afluxo de linfócitos e } \\
\text { histiócitos. }\end{array}$ \\
\hline & $\begin{array}{l}\text { 3. Em zona } 1,2 \text { e } 3 \text {, presença de vários focos de hepatocitólise } \\
\text { para os quais afluem mononucleares. }\end{array}$ \\
\hline & $\begin{array}{l}\text { 4. Veias hepáticas terminais de paredes finas ou com focos de } \\
\text { fibrose. }\end{array}$ \\
\hline & 5. Presença de colapsos focais da trama de reticulina. \\
\hline \multicolumn{2}{|r|}{ Conclusão } \\
\hline $\begin{array}{l}\text { Hepatite reacional: portite linfocitária crônica e } \\
\text { lobular multifocal } \\
\text { Hiperplasia kupferiana } \\
\text { Ausência de fibrose portal e lobular }\end{array}$ & $\begin{array}{l}\text { Colapsos focais da trama reticular } \\
\text { Ausências de atipias celulares }\end{array}$ \\
\hline
\end{tabular}

Quadro 2. Exame anatomopatológico do fígado do animal submetido à 2,0 g/kg i.p. de FAF de Cissus sicyoides.

\begin{tabular}{|c|c|}
\hline \multicolumn{2}{|c|}{ Órgão: Fígado } \\
\hline \multicolumn{2}{|c|}{$\begin{array}{l}\text { Material: Fragmentos teciduais, irregulares de fígado } \\
\text { Medida: O maior mede } 2,0 \times 0,3 \mathrm{~cm} \text {; o menor mede } 0,4 \times 0,1 \mathrm{~cm} \text {. }\end{array}$} \\
\hline Macroscopia & Microscopia \\
\hline $\begin{array}{l}\text { A superfície externa do material examinado é lisa, } \\
\text { cinza. }\end{array}$ & 1. Arquitetura do tipo lobular. \\
\hline \multirow{6}{*}{$\begin{array}{l}\text { Superfície de corte compacta. Seções representativas } \\
\text { encaminhadas para exame são submetidas às seguintes } \\
\text { colorações: hematoxilina-eosina, tricrômico de } \\
\text { Masson, picro-sirius e método de Gordon e Sweet } \\
\text { (reticulina), além de Perls, para detecção de } \\
\text { sobrecarga férrica. }\end{array}$} & $\begin{array}{l}\text { 2. Múltiplos focos de hapatocitólise, associados a infiltrado } \\
\text { linfocitário e histiocitário, em topografia periportal e lobular. }\end{array}$ \\
\hline & 3. Presença de colapsos focais da trama de fibras reticulares. \\
\hline & 4. Hiperplasia kupferiana \\
\hline & $\begin{array}{l}\text { 5. Espaços portais pequenos ás vezes, apresentando } \\
\text { mobilização leucocitária, mononuclear. }\end{array}$ \\
\hline & 6. Tríades vásculo biliares sem particularidades histológicas. \\
\hline & \begin{tabular}{|llllll}
$\begin{array}{l}\text { 7. Presença de exsudato inflamatório misto em seio } \\
\text { subcapsular }\end{array}$ & & & \\
\end{tabular} \\
\hline \multicolumn{2}{|c|}{ Conclusão } \\
\hline $\begin{array}{l}\text { Hepatite reacional: portite linfocitária crônica, } \\
\text { com necrose lobular multifocal } \\
\text { Hiperplasia kupferiana }\end{array}$ & $\begin{array}{l}\text { Colapsos focais da trama reticular } \\
\text { Ausências de atipias celulares }\end{array}$ \\
\hline Ausência de fibrose portal e lobular & Subcapsulite reacional \\
\hline
\end{tabular}




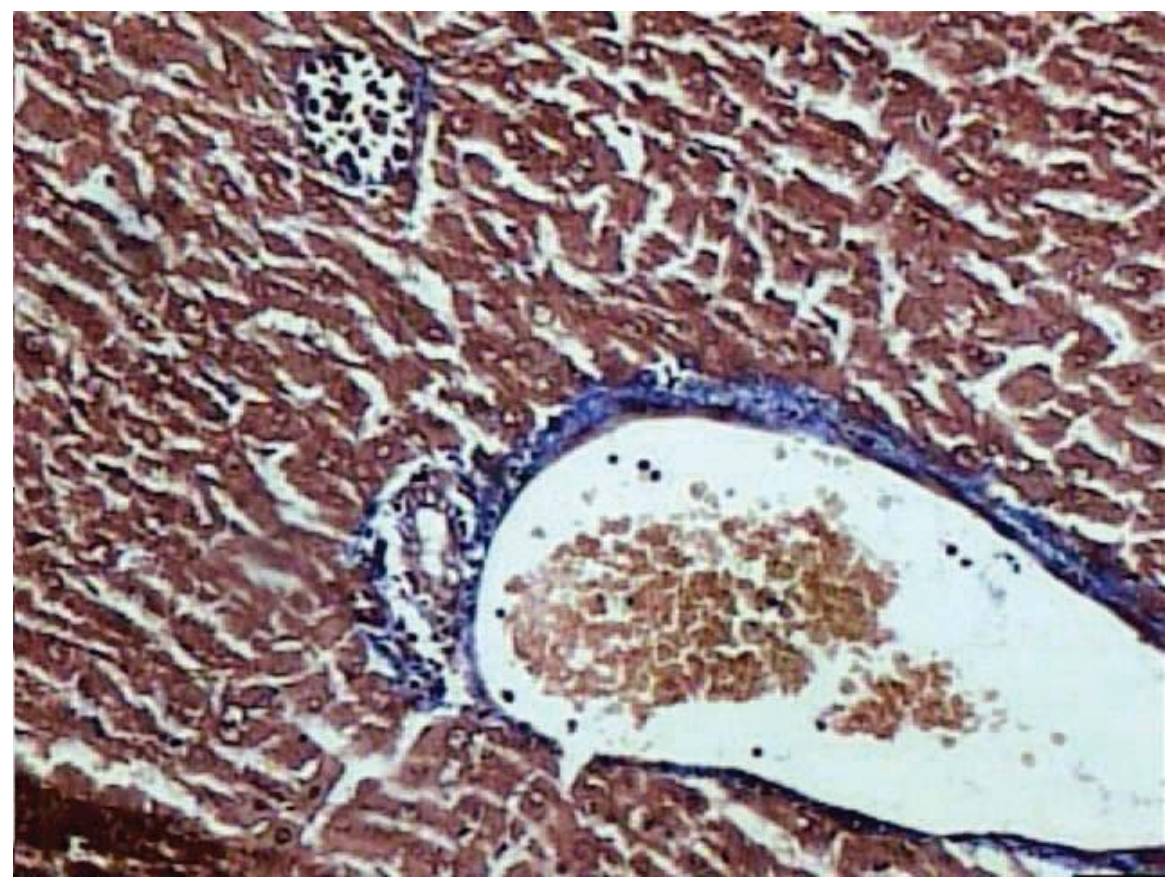

Figura 1. Fígado: Arquitetura parenquimatosa lobular. Hepatócitos distribuídos em traves paralelas e radiais, com relação a veia hepática terminal (VHT). (Colorações HematoxilinaEosina e Masson).

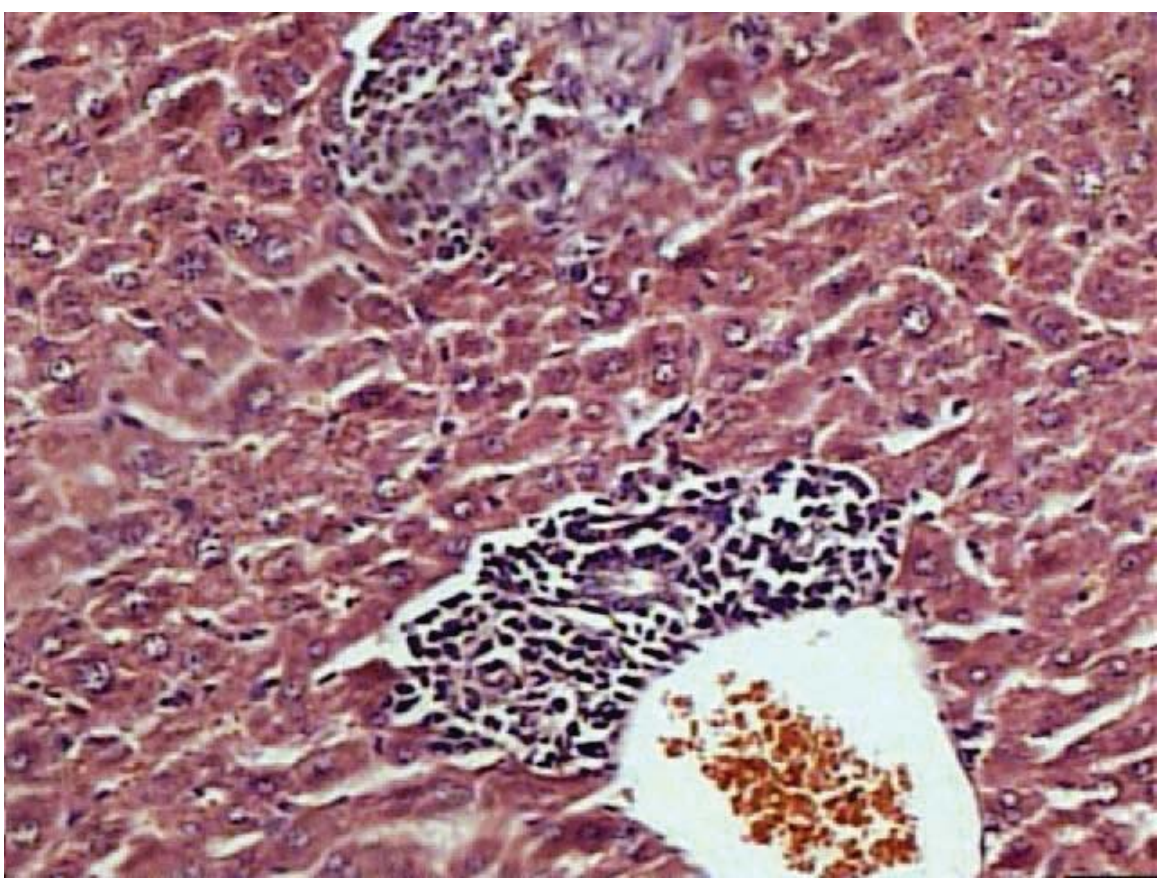

Figura 2. Fígado: Reação inflamatória mononuclear também é vista em alguns espaços portais (portite linfocitária). (Coloração Hematoxilina-Eosina).

Os efeitos tóxicos de substâncias podem atingir todos os sistemas e órgãos, porém é o fígado que paga o maior tributo, provavelmente devido a dois fatores importantes: a) a sua posição anatômica, que o torna mais vulnerável e, b) o seu próprio determinismo funcional, que condiciona maior concentração celular, não apenas dos compostos a serem transformados, como também dos metabólitos resultantes (Mendes, 1988).

Os pulmões representaram também substrato importante, para os efeitos de FAF neste estudo experimental. Apresentando uma discreta congestão vênulo-capilar para à dose $(5 \mathrm{~g} / \mathrm{kg})$ via oral e hiperplasia linfóide, folicular, peribronquiolar, focal para à dose (2 $\mathrm{g} / \mathrm{kg}$ ) via intraperitoneal. 


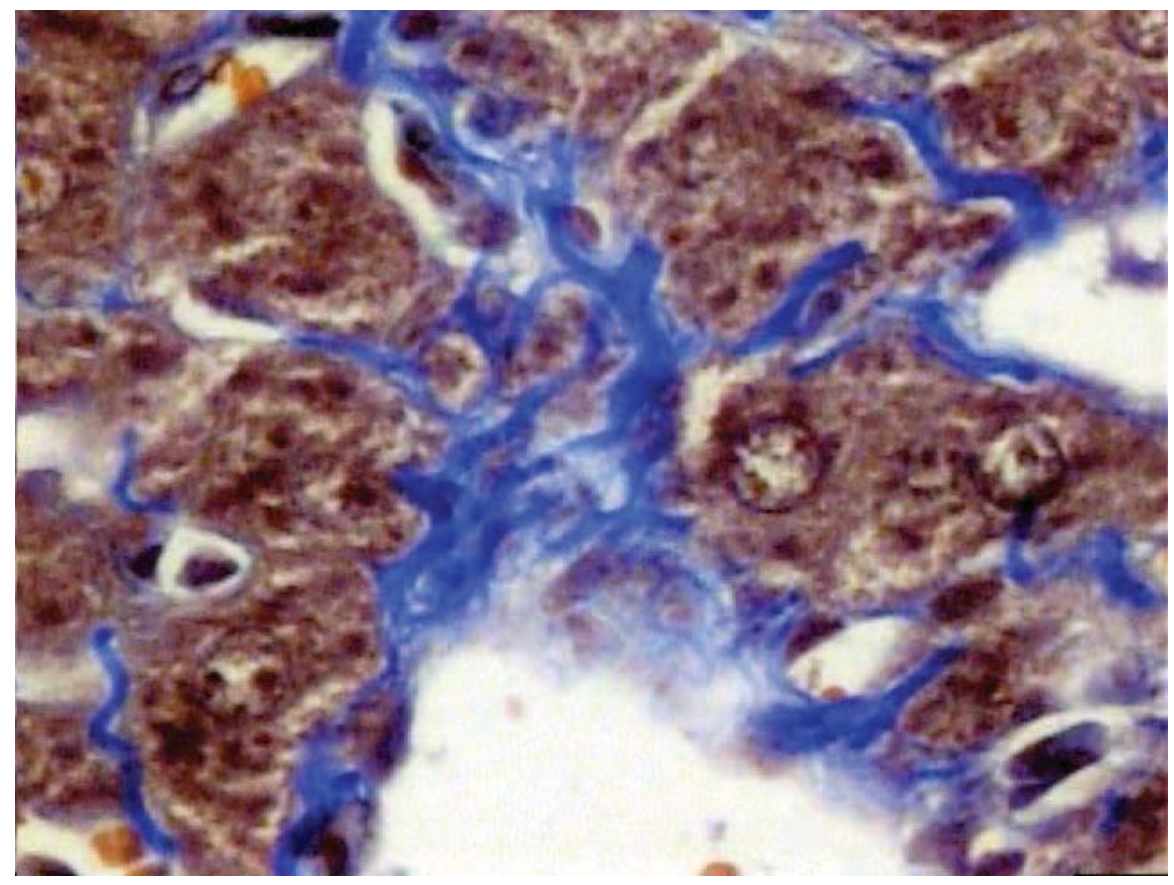

Figura 3. Fígado: Espessamentos colagênicos focais da parede da veia hepática terminal. Discreta fibrose sinusoidal, perivenular. (Coloração Masson).

O mais importante axioma da toxicologia é "a dose faz o veneno", ou seja, qualquer substância química pode ser tóxica se a dose ou exposição se tornar suficientemente altas, de modo que as alterações observadas foram atribuídas as altas doses utilizadas (Sipes; Dart, 1997; Diniz, 2000).

Assim, os dados obtidos na investigação de toxicidade aguda, avaliação das propriedades tóxicas que se produzem dentro de um curto período de tempo, com administração de uma dose única com FAF, demonstraram que nos camundongos Swiss, as doses máximas exigidas nos experimentos, não induziram mortes, mas evidenciaram alterações hepática e pulmonar, provavelmente decorrentes da administração de doses suficientemente altas para tal (dose 1.111 vezes a dose de uso popular $(4,5 \mathrm{mg} / \mathrm{kg})$, por via oral e dose 444 vezes a dose de uso popular, por via intraperitoneal).

\section{CONCLUSÃO}

O estudo toxicológico pré-clínico agudo em camundongos Swiss, tratados com as doses de $5,0 \mathrm{~g} / \mathrm{kg}$ via oral e $2,0 \mathrm{~g} / \mathrm{kg}$ via intraperitoneal da fração aquosa das folhas de Cissus sicyoides, demonstrou que o produto avaliado possui toxicidade aguda relativamente baixa, evidenciada pelos valores de $\mathrm{DL}_{50}$ superiores a $5,0 \mathrm{~g} /$ $\mathrm{kg}$ v.o. e $2,0 \mathrm{~g} / \mathrm{kg}$ i.p. e pelo fato de que as alterações hepáticas evidenciadas podem ser atribuídas aos valores relativamente altos das doses administradas.

\section{REFERÊNCIAS}

Anvisa 1999a. Agência Nacional de Vigilância Sanitária. Resolução - no17 - Diretrizes básicas para avaliação de risco e segurança dos alimentos, de 30/04/99. Diário Oficial da República Federativa do Brasil, Poder Executivo, Brasília, DF, republicada em 03/12/99.

Anvisa 1999b. Agência Nacional de Vigilância Sanitária. Resolução - oo18 - Diretrizes básicas para análise e comprovação de propriedades funcionais e ou de saúde alegadas em rotulagem de alimentos, de 30/04/99. Diário Oficial da República Federativa do Brasil, Poder Executivo, Brasília, DF, republicada em $03 / 12 / 99$.

Anvisa 1999c. Agência Nacional de Vigilância Sanitária. Resolução - no19 - Regulamento de procedimentos para registro de alimento com alegação de propriedades funcionais e ou de saúde em sua rotulagem, de 30/04/99. Diário Oficial da República Federativa do Brasil, Poder Executivo, Brasília, DF, republicada em 10/12/99.

Anvisa 2000. Agência Nacional de Vigilância Sanitária. Resolução - RDC no 17 de 24/02/2000. Diário Oficial da República Federativa do Brasil, Poder Executivo, Brasília, DF, 25 de fevereiro de 2000.

Angelucci E, Mantovani DMB 1986. Minerais em alimentos: manual técnico. Campinas: ITAL/SBCTA, 131p.

Aroch I, Srebro H, Shpigel NY 1999. Serum electrolyte concentrations in bitches with eclampsia. Vet Rec 145: 318-320

Bacha WJ, Wood LM 1990. Color atlas of veterinary histology. Ed. Lea \& Febiger. 269p.

Barbosa WLR, Santos WRA, Pinto LN, Tavares ICC 2002. Flavonóides de Cissus verticillata e a atividade 
hipoglicemiante do chá de suas folhas. Rev Bras Farmacogn 12(Supl.): 13-15.

Barbosa-Filho JM, Vasconcelos THC, Alencar AA, Batista LM, Oliveira RAG, Guedes DN, Falcão HS, Moura MD, Diniz MFFM, Modesto-Filho J 2005. Plants and their active constituents from South, Central, and North America with hypoglycemic activity. Rev Bras Farmacogn 15: 392-413.

Barbosa-Filho JM, Medeiros KCP, Diniz MFFM, Batista LM, Athayde-Filho PF, Silva MS, Cunha EVL, Almeida JRGS, Quintans-Júnior LJ 2006. Natural products inhibitors of the enzyme acetylcholinesterase. Rev Bras Farmacogn 16: 258-285.

Beltrame FL, Sartoretto JL, Bazotte RB, Cuman RN, Cortez DAG 2001. Estudo fitoquímico e avaliação do potencial antidiabético do Cissus sicyoides L. (Vitaceae). Quim Nova 24: 783-785.

Correa MP 1926. Dicionário das plantas úteis do Brasil. Imprensa Nacional; Rio de Janeiro, Vol. IV, p 130.

Diniz MFM 2000. Ensaios toxicológicos pré-clínicos com as folhas de Cissampelos sympodialis Eichl. Tese (Doutorado em Farmacologia) Universidade Federal da Paraíba, João Pessoa.

Litchfield LTJR, Wilcoxon F 1949. A simplified method of evaluation dose-effect experiments. $J$ Pharm Exp Ther 19: 388-397,

Mendes FT 1988. Fígado e drogas. In: Dani, R, Castro, LP. Gastroenterologia clínica. $2^{\text {a }}$ ed. Rio de Janeiro: Guanabara Koogan, cap. 80, p.1035-1042.

Miller O, Gonçalves RR 1999. Laboratório para o clínico. $8^{\text {a }}$ edição. São Paulo: Editora Arheneu, p.607.

Murthy KNC, Vanitha A, Swamy MM, Ravishankar GA 2003. Antioxidant and antimicrobial activity of Cissus quadrangularis L. J Med Food 6: 99-105.

Pepato MT, Keller E, Baviera AM, Lorenzoni A, Brunetti I 2000. Avaliação do efeito tóxico de Cissus sicyoides em ratos normais e diabéticos. Anais do XXXV Congresso Brasileiro de Fisiologia. FESBE.

Pepato MT, Baviera AM, Vendramini RC, Perez MPMS, Kettelhutt IC, Brunetti IL 2003. Cissus sicyoides (princess vine) in the long-term treatment of streptozotocin-diabetic rats. Biotechnol Appl Biochem 37: 15-20.

Secretaria de Vigilância Sanitária 1996. Resolução no 116/96, Diário Oficial da República Federativa do Brasil. 12/08/1996, Seção I, Brasília - DF, p.15199.

Sharon A, Center DMV 1995. Avaliação bioquímica da função hepática no cão e no gato. In: Atualização terapêutica veterinária: pequenos animais. p.1166-1183.

Shirwaikar A, Khan S, S. Malini S. 2003. Antiosteoporotic effect of ethanol extract of Cissus quadrangularis Linn. on ovariectomized rat. J Ethnopharmcol 89: 245-250.

Silva GA, Almeida-Muradian LB, Akisue G, Ferro VO. 1996. Padronização dos extratos de Cissus sicyoides (Insulina vegetal) e identificação de carotenos. Rev Bras Farmacogn 5: 96-112.

Silva L, Oniki GH, Agripino DG, Moreno PRH, Young MCM, Mayworm MAS, Ladeira AM 2007. Biciclogermacreno, resveratrole atividade antifúngica em extratos de folhas de Cissus verticillata (L.) Nicolson \& Jarvis (Vitaceae). Rev Bras Farmacogn 17: 361-367.
Sipes IG, Dart RC 1997. Toxicologia. In: Brody TM. et al. Farmacologia humana - da molecular à clínica. Rio de Janeiro: Guanabara Koogan, p.740-749.

Vasconcelos THC, Diniz MFFM, Cezarino EL, Medeiros IA, Lima IMBD, Gadelha NRA 2002. Ensaios toxicológicos clínicos com as folhas de Cissus sicyoides L. (Vitaceae). Anais do X Encontro de Iniciação Cientifíca da UFPB, Ciências da Vida, Pró-Reitoria de Pós-Graduação e Pesquisa - João Pessoa: Editora Universitária, p.99. 